\title{
The first Danish family reported with an AQP5 mutation presenting diffuse non- epidermolytic palmoplantar keratoderma of Bothnian type, hyperhidrosis and frequent Corynebacterium infections: a case report
}

\author{
Anne Bruun Krøigård ${ }^{1,3^{*}}$, Liv Eline Hetland², Ole Clemmensen³ ${ }^{3}$ Diana C. Blaydon ${ }^{4}$, Jens Michael Hertz ${ }^{1}$ \\ and Anette Bygum²
}

\begin{abstract}
Background: An autosomal dominant form of diffuse non-epidermolytic palmoplantar keratoderma, palmoplantar keratoderma of Bothnian type, is caused by mutations in the AQP5 gene encoding the cell-membrane water channel protein aquaporin 5 leading to defective epidermal-water-barrier function in the epidermis of the palms and soles.

Case presentation: We report the first Danish family diagnosed with diffuse non-epidermolytic palmoplantar keratoderma of Bothnian type in which fourteen individuals are potentially affected. The proband, a 36-year-old male had since childhood been affected by pronounced hyperhidrosis of the palms and soles along with palmoplantar keratoderma. He reported a very distinctive feature of the disorder, aquagenic wrinkling, as he developed pronounced maceration of the skin with translucent white papules and a spongy appearance following exposure to water. The patient presented recurrent fungal infections, a wellknown feature of the condition, but also periodic worsening with pitted keratolysis and malodour due to bacterial infections.
\end{abstract}

Conclusions: Palmoplantar keratoderma of Bothnian type, which may be associated with hyperhidrosis, is frequently complicated by fungal infections and may be complicated by Corynebacterium infections.

Keywords: Diffuse non-epidermolytic palmoplantar keratoderma, Palmoplantar keratoderma of Bothnian type, Corynebacterium infection, AQP5 gene, Aquaporin 5, "Hand-in-the-bucket-sign", Aquagenic wrinkling

\section{Background}

Palmoplantar keratodermas (PPKs) comprise a clinically and genetically heterogenous group of hereditary disorders of the skin characterized by thickening of the stratum corneum of the palms and soles. In some cases the skin lesions are accompanied by associated diseases [1]. Based on the clinical presentation, PPKs are divided into four subtypes, including diffuse, punctate,

\footnotetext{
* Correspondence: anne.kroeigaard@rsyd.dk

'Department of Clinical Genetics, Odense University Hospital, Odense, Denmark

${ }^{3}$ Department of Clinical Pathology, Odense University Hospital, Odense, Denmark

Full list of author information is available at the end of the article
}

focal and striate PPK [2]. Diffuse PPK can be further subdivided histopathologically into epidermolytic and non-epidermolytic forms depending on the presence or absence of cytolysis in the upper spinous and granular layers of the epidermis. Diffuse non-epidermolytic PPK of Bothnian type (PPKB), is not accompanied by associated diseases and was initially described in two Swedish families [3]. PPKB (OMIM 600231) is autosomal dominantly inherited and initial studies linked the underlying gene defect to chromosome 12q11-q13 [3, 4]. In 2013, the genetic cause of PPKB was established as due to mutations in the $A Q P 5$ gene, located at 12q13.12 [5]. In the 
northernmost region of Sweden the condition is relatively common with a reported prevalence of $0.3-0.55 \%$ [3].

The clinical presentation of PPKB include diffuse palmoplantar hyperkeratosis with a yellowish tint over the whole of the palms and soles and acral hyperhidrosis [5]. The symptoms usually presents in early childhood, in some cases as young as three months of age [6]. Secondary dermatophyte infections are frequent. A distinctive feature of this type of PPK is a whitish spongy appearance of the skin following exposure to water. The phenotypic apperarence of PPKB varies and in some individuals, clinical signs of the condition are only seen after exposure to water [5].
In this study, we present the first Danish family with autosomal dominant PPKB, caused by a heterozygous p.Arg188Cys mutation in the $A Q P 5$ gene. The proband presented with hyperhidrosis and subsequent complications caused by superinfections with dermatophytes and Corynebacteria.

\section{Case Presentation}

A large four-generation Caucasian family was ascertained, including 14 affected individuals, as seen in Fig. 1. The proband was a 36-year-old male, affected since childhood by palmoplantar keratoderma, pronounced hyperhidrosis and recurrent secondary dermatophyte

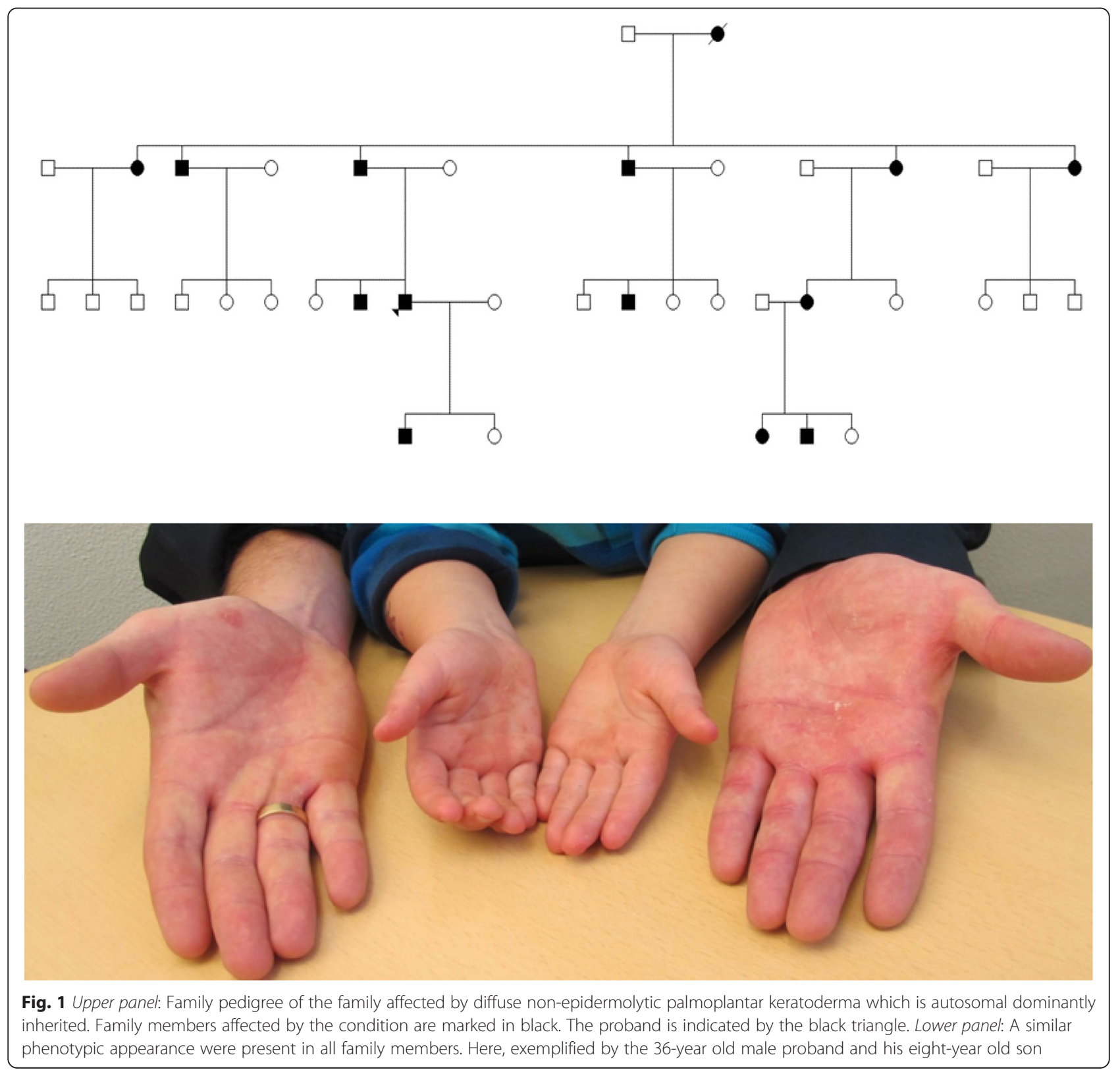



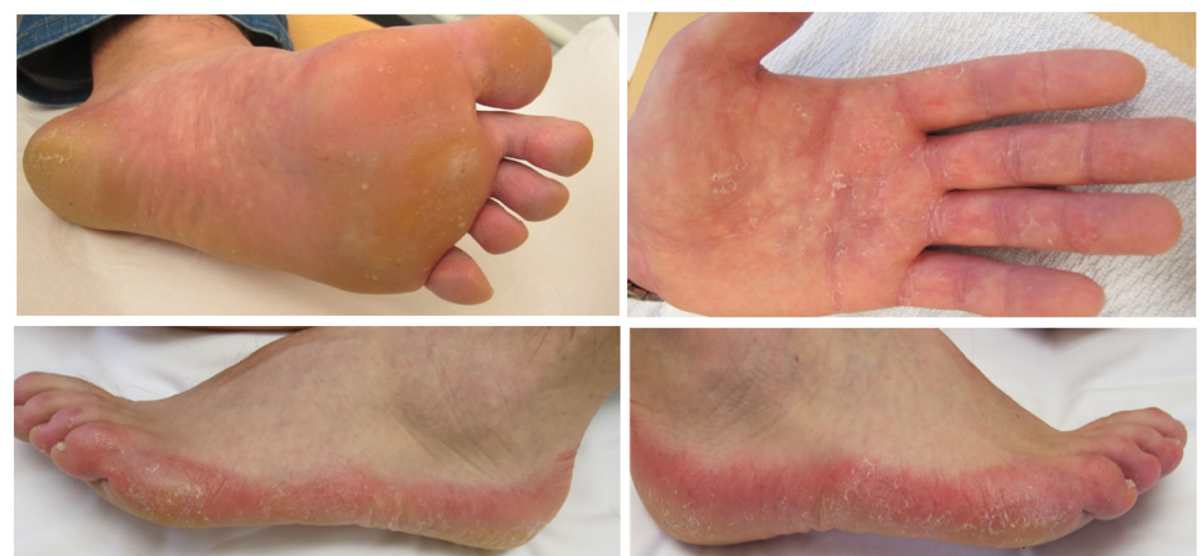

Fig. 2 Phenotypic appearance of the 36-year old male proband affected by diffuse nonepidermolytic palmoplantar keratoderma. The palmar and plantar skin was affected by yellow tinted keratoderma, pitted keratolysis and erythrokeratotic plaques with a clear demarcation to normal skin on the dorsum of the hands and feet. Sparing of the skin of the arches were seen on planta pedis

infections on affected skin. Examination of the skin revealed a mild, yellow tinted diffuse keratoderma of palms and soles, pitted keratolysis and erythematous keratotic plaques with a clear demarcation on the margins of the hands and feet, as seen in Fig. 2. Sparing of the skin was seen in the arches of the planta pedis. Physical examination was non-contributory. The skin lesions were periodically foul-smelling and the patient suffered from recurrent tinea pedis and onychomycosis caused by dermatophyte infections. Thirteen additional family members presented with a similar phenotype. Initially, the diagnosis epidermolytic palmoplantar keratoderma was suspected. Mutational screening of KRT1, KRT9 and KRT16 genes provided negative results.

The biopsy showed a markedly thickened stratum corneum with a prominent stratum granulosum and a moderate acanthosis, as seen in Fig. 3. The acrosyringial ducts were remarkably dilated as well in the epidermis as in the stratum corneum. The distal part of the eccrine ducts in the papillary dermis, however, were without recognizable dilatation. Focally, changes like those seen in miliaria rubra with spongiosis and exocytosis of lymphocytes around the intraepidermal acrosyringial channels were noted. The lymphocytic infiltration extended

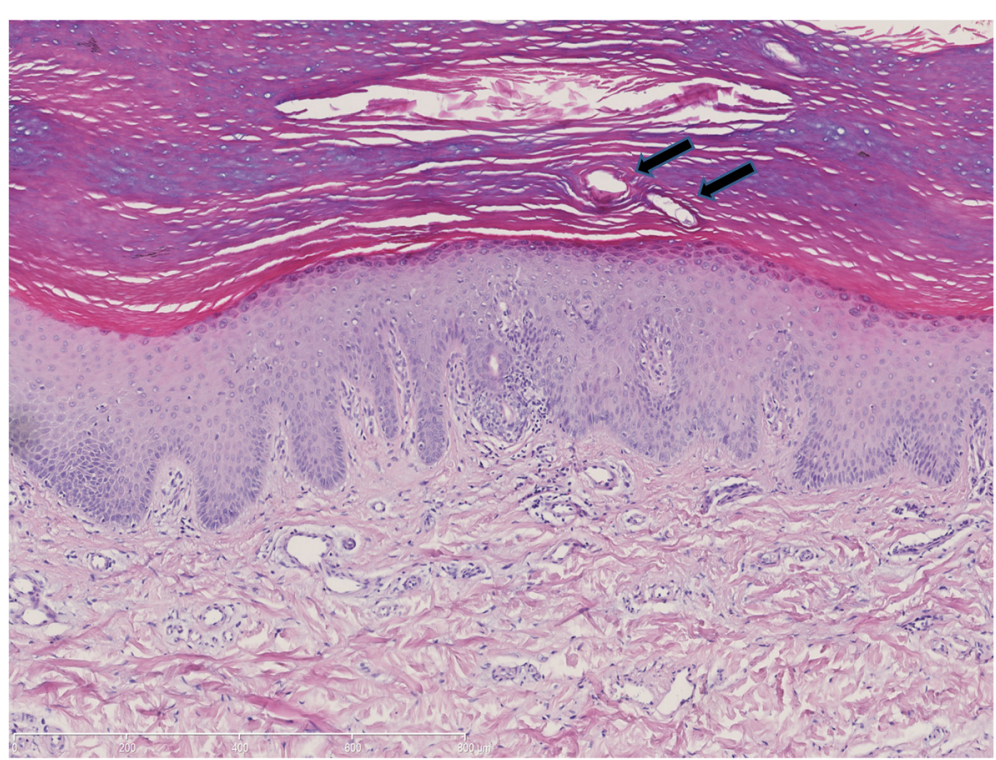

Fig. 3 Punch biopsy from the foot, hematoxylin and eosin stained. The stratum corneum is thickened with epidermal acanthosis. The acrosyringeal intracorneal ducts are dilated (arrows), and focally miliaria rubra like changes with spongiosis of the eccrine duct and lymphocytic infiltration around the duct is seen 
into the papillary dermis around and into the distal parts of the dermal sweat ducts, but the hyperkeratotic plugging, often seen in miliaria rubra above the spongiosis, was not present in this biopsy. The proximal part of the sweat ducts and the secretory coil appeared normal. Spores and hyphae indicating a dermatophytosis were demonstrated in the stratum corneum. Corynebacteria, however, were not seen.

Water immersion test revealed aquagenic wrinkling, also known as "hand-in-the-bucket-sign", as translucent white papules and a whitish spongy appearance due to swelling of the stratum corneum was observed after three minutes exposure to water, as seen in Fig. 4. The aquagenic wrinkling lead to suspicion of transient reactive papulotranslucent acrokeratoderma [7] or aquagenic syringeal acrokeratoderma [8] but the proper diagnosis was not established until the report by Blaydon et al. [5] raised the suspicion of PPKB.

\section{Genetics}

Following informed consent, genomic DNA purified from a blood sample from the proband was analyzed using bidirectional Sanger sequencing of the AQP5 gene. Genetic test results available in Additional file 1: Figure S1. The patient was heterozygous for a missense mutation in the AQP5 gene, c.562C $>\mathrm{T}$, (p.Arg188Cys). This exact mutation is previously described to cause autosomal dominant PPKB [5]. The probands eight year old son
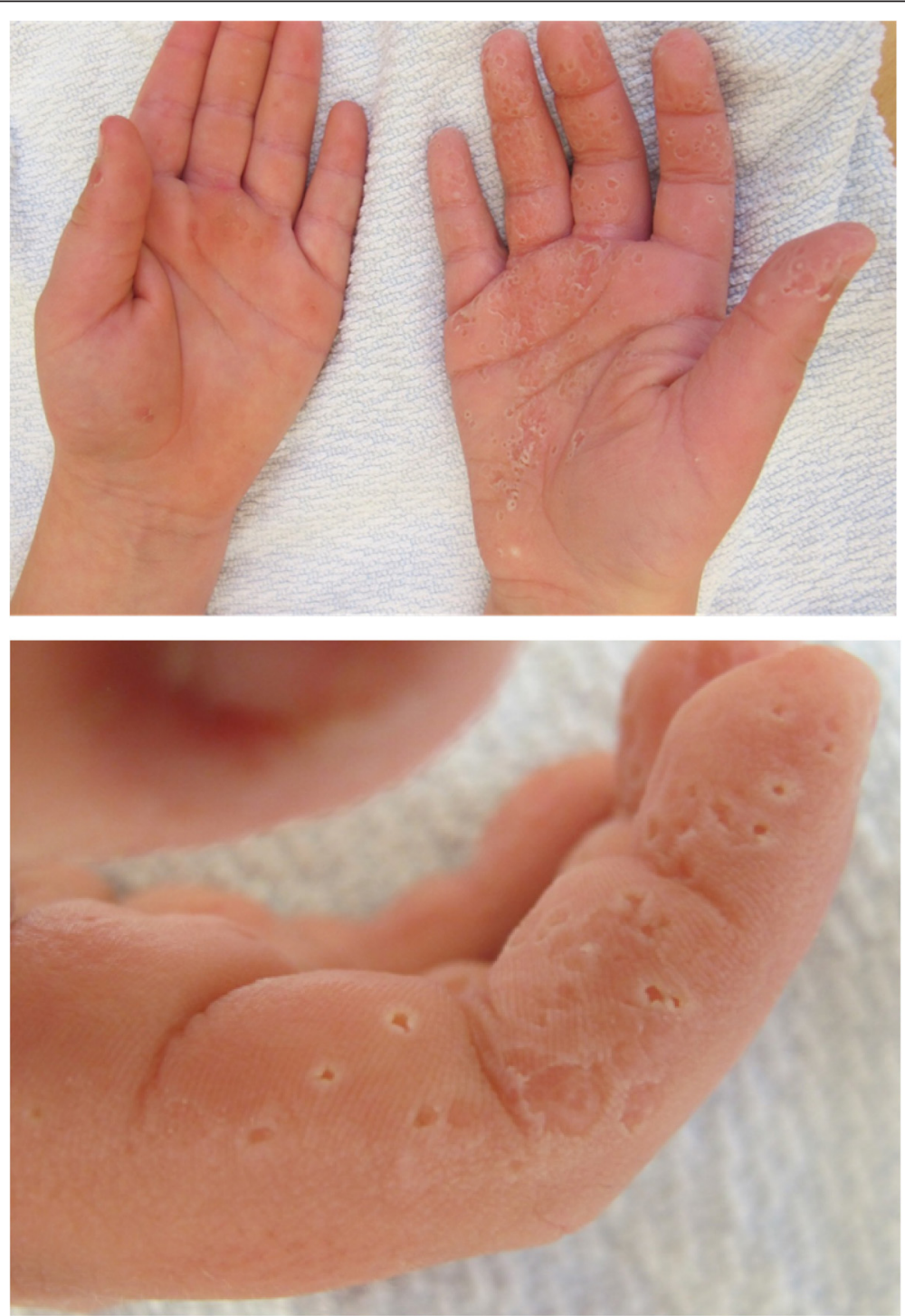

Fig. 4 Aquagenic wrinkling, also known as "hand-in-the-bucket-sign" revealed by water immersion test. Upper panel: Palmar phenotype after water immersion test of only the right hand. After three minutes in water, the palmar skin on the right hand was clearly affected by translucent white papules and a whitish spongy appearance, compared to the left side which had not been exposed to water. Lower panel: Spongy appearance on affected skin due to swelling of the stratum corneum after three minutes exposure to water 
was found to be heterozygous for the same $A Q P 5$ mutation. The remaining affected family members have not consented to genetic testing.

\section{Superinfection with corynebacterium}

Interestingly, the dermatophyte infection of the plantar skin was complicated by a superinfection with Corynebacterium. Examination in Woods light showed massive coral red fluorescence in interdigital areas of planta pedis, as seen in Fig. 5. The Corynebacterium infection was treated with clindamycin and chlorhexidine with satisfactory effect.

\section{Discussion}

We present the first Danish family diagnosed with autosomal dominant PPKB resulting from c.562C $>\mathrm{T}$, p.Arg188Cys in the AQP5 gene. According to the $\mathrm{Hu}-$ man Gene Mutation Database a total of six missense mutations in the AQP5 gene associated with PPKB have been reported to this date [9] in families of Swedish, British, Scottish and Han Chinese descent [2, 5]. The condition has also been reported in a patient from India [10].

The AQP5 gene encodes aquaporin 5, a cell-membrane protein that allows osmotic movement of water across the cell membrane independently of solute transport [11]. Aquaporin 5 is situated in the apical plasma membrane of cells of the sweat glands, salivary glands, lacrimal gland, lung, cornea [11] and in the palmoplantar epidermis [5]. The study by Blaydon et al. reported that the expression of AQP5 in the stratum granulosum was not affected by mutations in the gene [5]. Hence, at the genetic level, mutations associated with PPKB most likely induce increased expression of the gene or excert a gain-of-function effect leading to accelerated keratinocyte water uptake. This likely explains the swelling of the stratum corneum of the epidermis following water exposure.

The notion of increased function of aquaporin 5 in PPKB is supported by the fact that immunolocalization experiments involving Sjögrens syndrome, which is characterized by hypohidrosis, revealed reduced expression of AQP5 in sweat glands from patients with Sjögrens compared to normal skin [12]. Thus, the hypohidrosis observed in Sjögrens syndrome may result from reduced expression of AQP5 and correspondingly, the hyperhidrosis of PPKB may result from an increased function of the gene product. From a clinical perspective, the hyperhidrosis characterizing PРКB entails notable discomfort to the patient and most likely is a contributing factor to the recurrent Corynebacterium infections. Conversely, it has recently been hypothesized that hyperhidrosis may be exacerbated by bacterial infection [13].

The histopathologic unspecific features of hyperkeratosis and acanthosis are in agreement with the findings in PPKB. In addition, however, we found focal changes around the intraepidermal sweat ducts as seen in conditions like miliaria rubra, also known as heat rash. To our knowledge, this has not been described in PPKB. It is possible that the biopsy was taken during an episode of hyperhidrosis, but the inflammatory changes of miliaria rubra typically do not accompany ordinary hyperhidrosis. It seems likely that the changes of stratum corneum in PРКB may obstruct the sweating and induce the miliaria rubra like changes. It certainly would be of interest to study biopsies from larger series of PPKB to assess the specificity of this feature.

Whether the pitted keratolysis results from the palmoplantar keratoderma, the hyperhidrosis or the Corynebacterium infection is not established. Treatment options for PPKB include salicylic acid 4-6 \% in petrolatum, urea- or salicylic acid-containing creams and

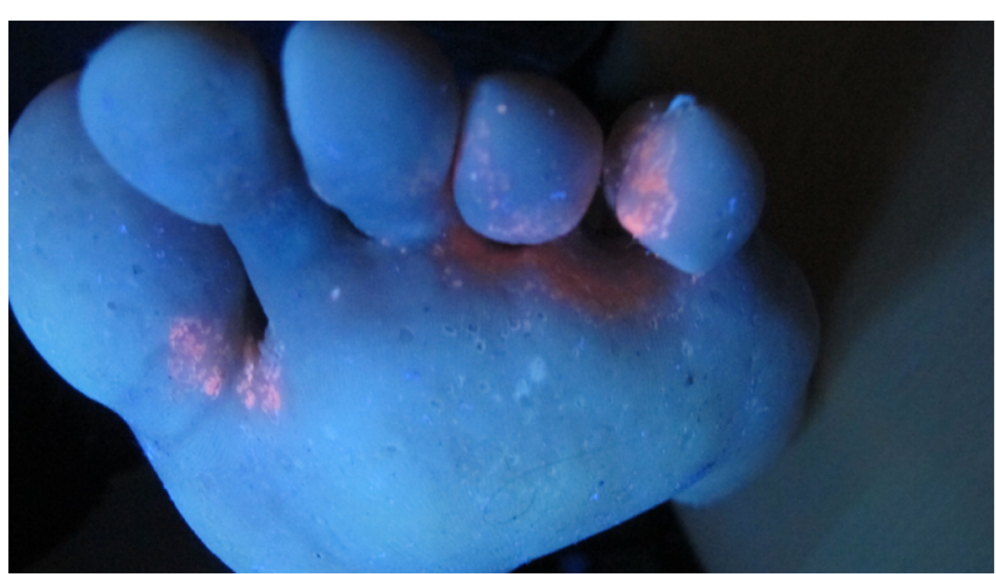

Fig. 5 Examination of the plantar skin in Woods light showed massive coral red fluorescence in interdigital areas representing a superinfection with Corynebacterium 
lotions and avoidance of too much moisture. The hyperhidrosis may respond to aluminum chloridecontaining products or other therapeutic options of hyperhidrosis. Treatment of secondary dermatophyte infections and superinfections with bacteria like Corynebacterium is of extreme importance for optimal patient care.

The "hand-in-the-bucket-sign" is not pathonomonic of PPKB as aquagenic wrinkling of the palms following exposure to water is observed in several conditions including transient reactive papulotranslucent acrokeratoderma and aquagenic syringeal acrokeratoderma and is suggested to be more frequent than previously assumed [14]. In accordance with the PPKB genotype-phenotype correlation, aberrant expression of aquaporin 5 has been reported to be associated with aquagenic wrinkling of the palms [15]. Conversely, aquagenic keratoderma has also been suggested to be associated with mutations in the CFTR gene involved in cystic fibrosis $[16,17]$.

\section{Conclusions}

Though increasing evidence suggests a gain-of-function effect of AQP5 mutations related to PPKB, the exact mechanisms by which mutations in AQP5 lead to defective epidermal-water-barrier function remains to be elucidated. Conclusively, we have yet to arrive at a final understanding and characterization of conditions of disturbed water permeability of the palmoplantar epidermal cells.

The present manuscript adds a Danish family with PPKB to the literature and emphasizes that Corynebacterium superinfections may complicate the condition.

\section{Additional file}

Additional file 1: Figure S1. Genetic test results of the proband. Bidirectional Sanger sequencing of the AQP5 gene. (PDF 617 kb)

\section{Abbreviations}

PPK, Palmoplantar keratoderma; PPKB, Diffuse non-epidermolytic PPK of Bothnian type

\section{Acknowledgements}

The authors thank professor Maurice Van Steensel for fruitful advice in the diagnostic process and the family for participating in the study.

\section{Authors' contributions}

$A B$ and LEH were the treating physicians and performed the clinical evaluation. OC was the pathologist on the case and performed the histopathological examination of skin biopsies. JMH and DCB were responsible for genetic testing and genetic counselling. ABK wrote the manuscript. All authors read and approved the final manuscript.

\section{Competing interests}

The authors declare that they have no competing interests.

\section{Consent for publication}

Written informed consent was obtained from the patient for publication of this case report and any accompanying images.

\section{Author details}

'Department of Clinical Genetics, Odense University Hospital, Odense, Denmark. ${ }^{2}$ Department of Dermatology and Allergy Centre, Odense University Hospital, Odense, Denmark. ${ }^{3}$ Department of Clinical Pathology, Odense University Hospital, Odense, Denmark. ${ }^{4}$ Centre for Cell Biology and Cutaneous Research, Blizard Institute, Bart and the London School of Medicine and Dentistry, Queen Mary University of London, London, UK.

Received: 20 January 2016 Accepted: 23 May 2016

Published online: 03 June 2016

\section{References}

1. Itin PH, Fistarol SK. Palmoplantar keratodermas. Clin Dermatol. 2005;23:15-22.

2. Cao X, Yin J, Wang H, Zhao J, Zhang J, Dai L, Zhang J, Jiang H, Lin Z, Yang Y. Mutation in AQP5, encoding aquaporin 5, causes palmoplantar keratoderma Bothnia type. J Invest Dermatol. 2014;134:284-7.

3. Lind L, Lundström A, Hofer PA, Holmgren G. The gene for diffuse palmoplantar keratoderma of the type found in northern Sweden is localized to chromosome 12q11-q13. Hum Mol Genet. 1994;3:1789-93.

4. Kelsell DP, Stevens HP, Ratnavel R, Bryant SP, Bishop DT, Leigh IM, Spurr NK. Genetic linkage studies in non-epidermolytic palmoplantar keratoderma: evidence for heterogeneity. Hum Mol Genet. 1995;4:1021-5.

5. Blaydon DC, Lind LK, Plagnol V, Linton KJ, Smith FJD, Wilson NJ, McLean WHI, Munro CS, South AP, Leigh IM, O'Toole EA, Lundström A, Kelsell DP. Mutations in AQP5, encoding a water-channel protein, cause autosomaldominant diffuse nonepidermolytic palmoplantar keratoderma. Am J Hum Genet. 2013;93:330-5.

6. Abdul-Wahab A, Takeichi T, Liu L, Lomas D, Hughes B, Akiyama M, et al. Autosomal dominant diffuse non-epidermolytic palmoplantar keratoderma due to a recurrent mutation in aquaporin-5. Br J Dermatol. 2015;174:430-2.

7. Adişen E, Karaca F, Gürer MA. Transient reactive papulotranslucent acrokeratoderma in a 50-year-old woman: case report and review of the literature. Am J Clin Dermatol. 2008:9:404-9.

8. Baldwin BT, Prakash A, Fenske NA, Messina JL. Aquagenic syringeal acrokeratoderma: report of a case with histologic findings. J Am Acad Dermatol. 2006;54:899-902.

9. Stenson PD, Mort M, Ball EV, Shaw K, Phillips A, Cooper DN. The Human Gene Mutation Database: building a comprehensive mutation repository for clinical and molecular genetics, diagnostic testing and personalized genomic medicine. Hum Genet. 2014;133:1-9.

10. Das A, Kumar D, Das NK. Diffuse non-epidermolytic palmoplantar keratoderma. Indian Pediatr. 2013;50:979.

11. King LS, Kozono D, Agre P. From structure to disease: the evolving tale of aquaporin biology. Nat Rev Mol Cell Biol. 2004;5:687-98.

12. lizuka T, Suzuki T, Nakano K, Sueki H. Immunolocalization of aquaporin-5 in normal human skin and hypohidrotic skin diseases. J Dermatol. 2012;39:344-9.

13. Pranteda G, Carlesimo M, Pranteda G, Abruzzese C, Grimaldi M, De Micco S, Muscianese M, Bottoni U. Pitted keratolysis, erythromycin, and hyperhidrosis. Dermatol Ther. 2014;27:101-4.

14. Ertürk-Özdemir E, Özcan D, Seçkin D. Acquired aquagenic syringeal acrokeratoderma: A case series of 10 patients. Australas J Dermatol. 2015;56:e43-5.

15. Kabashima K, Shimauchi T, Kobayashi M, Fukamachi S, Kawakami C, Ogata M, Kabashima R, Mori T, Ota T, Fukushima S, Hara-Chikuma M, Tokura Y. Aberrant aquaporin 5 expression in the sweat gland in aquagenic wrinkling of the palms. J Am Acad Dermatol. 2008;59(2 Suppl 1):S28-32.

16. D'Erme AM, Braggion C, de Martino M, Greco A. Aquagenic palmoplantar keratoderma: a sign of cystic fibrosis early in life. Int J Dermatol. 2014;53: e536-8

17. Rongioletti F, Tomasini C, Crovato F, Marchesi L. Aquagenic (pseudo) keratoderma: a clinical series with new pathological insights. $\mathrm{Br} J$ Dermatol. 2012;167:575-82. 